\title{
Fingerprint Technology and Sustainable Development
}

\author{
Assiya Utzhanova ${ }^{1}$
}

\begin{abstract}
This paper explores to what extent fingerprint technology is reliable in technical, social and environmental aspects. Beginning from its history, application in business to how it's modelled, statistical analysis on probability of mismatch, general overview of its effect on environmental sustainability, and attitudes towards fingerprint technology in society. Assessing the reliability of automated biometrics is important because of its application in business for security and user authentication purposes. It increases environmental awareness. Usage of fingerprint technology provides business opportunities that substitute traditional systems of credit card, written timeattendance records, and cash. It leads to sustainable development by using minimum resources; compared to other systems its application reduces production of plastic, paper consumption and contributes by energy and operational efficiency.

Statistical analysis of fingerprint technology in framework of binomial, normal and Poisson distributions strengthens the reliability of fingerprint system. Source of the minutiae points used to analyze fingerprint characteristics is image of personal fingerprint. Statistical tests show that the accuracy may be improved. If the number of minutiae points increases, the probability of mismatch: type 1 , type 2 errors decreases.

Real-time experiments tested efficiency of system and provided data for hypothesis tests that challenged uniqueness of fingerprints. Social and ethical issues related to fingerprint recognition system were analyzed by conducting survey. Insecure storage of fingerprint image implies risk to the privacy of stakeholders, thus the system is not completely reliable for wider application. Accurate system design and improvement of such imperfections will generate more trust by stakeholders that will guide further towards sustainable development.
\end{abstract}

Key words: The reliability of fingerprint technology in user authentication, its application in business and impact on environmental sustainability.

\section{Introduction to Biometrics}

Biometrics is a form of verification used for authentication purposes. As Roethenbaugh said, 'biometrics is a science which involves statistical analysis of biological characteristics' (Roethenbaugh, 1998). The first sign of biometrics was found in 500 B.C., when fingerprints were representing person's signature. In fact, Babylonian business transactions were recorded on clay tablets using the fingerprint. For Egyptians the biometrics was used to differentiate merchants of a good and bad reputation, as a result it measured the reliability of a person. In 1858 the first systematic hand image had been captured for identification purpose. Approximately 30 years later, Professor Galton developed a classification system for fingerprints. As a result, F. Galton, E. Henry and H. Faulds officially became the founders of a fingerprint as a tool for purposes of person 
identification (Hawthorne, 2009).

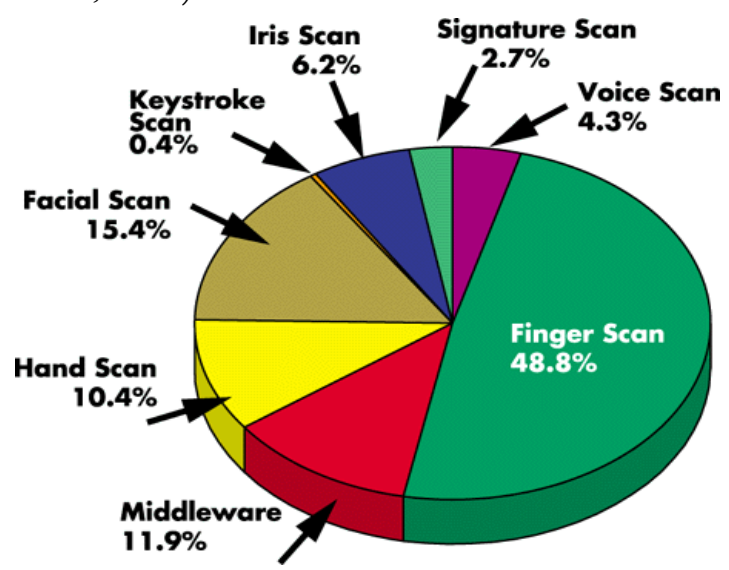

Figure 1. Percentage of types of Biometrics system sold on market (Biometrics Competitive Landscape, n. d.).

Figure 1 shows the percentage (48.8\%) of fingerprint scanners that is earned in the U.S. market sales. It is a dominant form of authentication compared to hand scan, facial scan, iris in the United States. It serves as the evidence for the increasing demand on fingerprint system by various businesses.

\subsection{Applications of Fingerprint Technology in Business}

Fingerprint scanner manufacturer Simprints offer legal identity for populations who are undermined and belong to rural areas in Asia and Africa through registering their identities through fingerprint technologies in government database (Rowe, 2016). Technology is designed for environment that requires wireless, low-cost innovations. In such way demographics adapt to and become part of the legal world.

Fingerprint scanners are also used by banking systems, ATMs as PIN. In order to increase efficiency, Japan is planning to test fingerprint payment system, where 2 fingers input on RFID scan can pay for any expenses or services within 300 firms in Japan (Chang, 2016). The fingerprints will be linked to credit card data and act as currency. Another application of such technology is genetic test. Using fingerprint pattern recognition techniques and big data analytics such test predicts areas of talents of individual, which provides opportunities for faster adaptation to environment and recognizing individual potential and deciding on career choice, abilities in science, sports, and personal characteristics (Genetic Test, 2014).

Sweden's Fingerprint Card System is planning to use fingerprints to pay for public transportation. Its market share has risen at 1600 percent $(4.1 \$$ bln $)$ within increasing demand of fingerprint technology (Chang, 2016). This type of IT system provides secure access to buildings, time-attendance records for small and large organizations, schools; substitutes passwords on laptops for confidential information. 


\section{Effect of Fingerprint Technology to Sustainability}

In environmental terms usage of fingerprint technology in these spheres leads to sustainable development. It is observed from an example of Biolink manufacturer. Production of fingerprint FTIR scanners complies with Restriction of Hazardous Substances standards. Infrared lights scan fingerprint, but it is not harmful because the rays are unseen by human eyes during scanning process. Manufacturer uses various metals and plastic in production that can be utilized. Organic glass is used for scanning process. Energy Consumption of terminal scanner is 1 Ampere/h; for USB scanner it is 150 milliampere/ $h$. This technology is environmentally friendly; it also minimizes the use of resources and does not consume vast amounts of energy. Its main purpose is to meet consumers' needs by allowing faster verification into different systems (operational efficiency) and providing physical and information security for stakeholders of particular environment.

\subsection{Reduction in Paper Consumption}

Biometrics requires electronic storage of information; therefore it reduces paper consumption heavily (Straub, 2016). The purpose of planting trees and forests is to provide oxygen, clean air and combat climate change. When humans waste tons of paper for short term usage, alternatively they could allow the tress to fully grow for future population, because trees are not renewable in short time. Any tree requires approximately 30 years to fully grow. An acre of fully grown tree provides 18 people with oxygen (Barinova, 2016). According to WWF, 120000 to 150000 square kilometers of forests are cut off every year (Barinova, 2016). When time-attendance system is managed by biometrics based time-clock software, all data is digitized and stored in the database rather than printed records. Application of fingerprint technology increases environmental awareness as it has the potential to save some forests from being cut, so they could increase the positive externalities such as oxygen for the environment. The use of fingerprints as currency in Japan reduces production of credit cards, plastic, and decreases demand on printing cash, which in turn saves more forests (Velandia, Katherine, 2012).

\subsection{Decrease in Production of Plastic}

6-17 billion plastic cards containing PVC are produced every year (Meet, 2011). PVC is common for credit cards too. The material is not recyclable, after the usage of card, as time passes only its size changes, making it problematic to ingest for creatures which confuse the colorful cards with food, such as birds and fishes in oceans.

Greenpeace explains that no other plastic is as harmful as PVC to human health and environment, yet its demand function is upward sloping (Meet, 2011). For security purposes repurposing old personalized cards is not safe way to recycle after their 3 years expiry date. Therefore methods alternative to plastic cards such as fingerprint authentication could work as a substitute technology to meet the consumer demand, saving the energy required for yearly production of banking cards. It can reduce health 
and environmental impacts of PVC as fingerprint is a biological characteristic, natural to human being that identifies person and is environmentally friendly. Fingerprint scanners are used for longer duration than magnetic cards, as fingerprint does not change over time and is extremely hard to be stolen (Zhang, 2000).

\section{Fingerprint Modeling. Minutiae Extraction Algorithm}

When a person puts his fingerprint (fig.2) on the scanner, the system creates a template in a database (fig. 3), where it stores not the fingerprint image, but the numerical value of the extracted minutiae points from the fingerprint (fig. 5). The next time person inputs their fingerprint onto the recognition hardware, the entered minutiae points are simply matched mathematically to the ones stored in the database, resulting in a binary image (fig. 5). Thus, this process is given in complex mathematic equations requiring vectors and coordinate geometry (fig. 4). The algorithm is as follows (Fingerprint

Technology

Overview, n.d.):

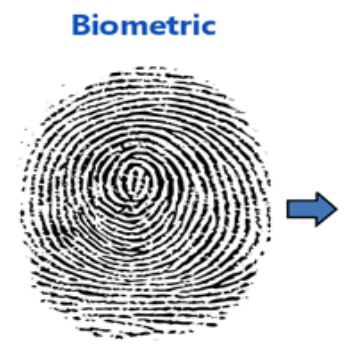

Minutia Points

Minutia Map

\section{Data Stream}
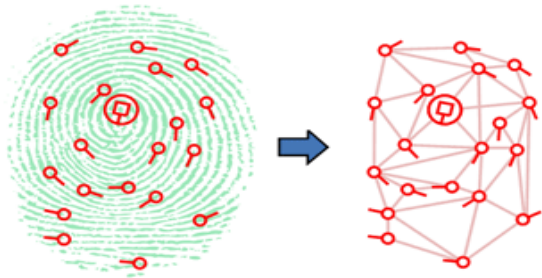

0101010001101000011010 0101110011001000000110 1001011100110010000001 1011100110111101110100 001110100011101010110 1101101000110101011 0001011011000110110001 1110010010000001100110 1101100101011100100111 0000011100100110100101 1011100111010000100000 0110010001100001011101 0001100001001011000010

fig. 2

fig. 3

fig. 4

fig. 5

In figure 4, pixel '1' indicates ridge and pixel 'O' indicates to the valley of the fingerprint. (Zhang, 2000)
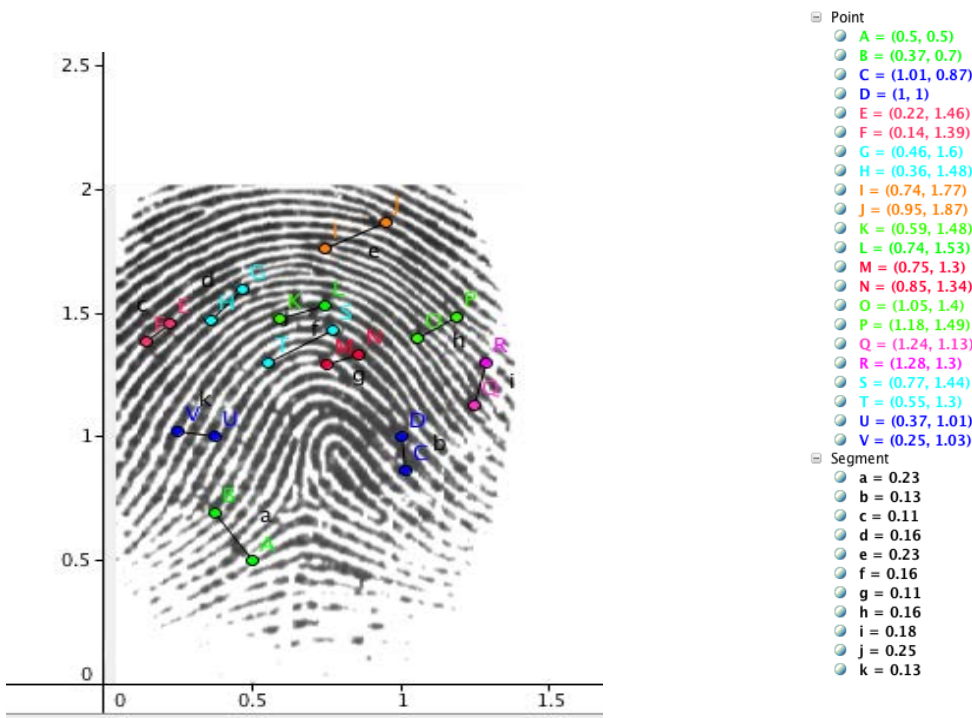

Figure 6 shows segments of minutiae points of individual fingerprint calculated using Geogebra. 
The skin on human fingers, especially palms and soles, demonstrates a pattern of ridges friction ridges. Light radiated by electromagnetic waves that belong to fingerprint scanners travels to the blood vessels, and sensor absorbs reradiated light in different patterns. The unique and changeless pattern which friction ridge possesses creates the fingerprint image (Kothavalte, Markworth, Sandhu, 2004). The ridges are not always continuous and a point where the ridge's curve dispatches in multiple directions is the imperfection called minutiae point.

Using GeoGebra the distance between 2 closest minutiae points was taken to be a segment. 11 segments show 22 minutiae points extracted from the finger, which are perceived to be unique physical characteristics of individual (Zhang, 2000). The next stage in the fingerprint technology algorithm is converting analogue data into digital form. It is done using binary system notation. Afterwards, system performs decision making that results match/no match.

\subsection{Statistical Analysis of Fingerprint Recognition System} distribution.

Problem: Find probability that 2 fingerprints are equivalent, given Gaussian Conditions: 2 fingerprints are independent events; $\mathrm{X}$ is a random variable that represents a number of minutiae matches. $X \sim N\left(\mu, \delta^{2}\right)$. Many forensic domains assume Gaussian (normal) distribution for fingerprints matching process (Su, Srihari, 2010). $E X=0.504$; $\mathrm{P}(\mathrm{X}<4)=\frac{1149}{50000}=0.029$; Data is extrapolated from a study with 50000 samples of fingerprint repetitions. As Forensic guidelines state when two fingerprints have minimum of 12 numbers of minutiae matches, they are said to come from one finger (Yang-Jea, Govindaraju, 2005).

$$
\left\{X \mid \begin{array}{c}
x \geq 12 ; 2 \text { fingerprints are equivalent } \\
x<12 ; 2 \text { fingerprints are not equivalent }
\end{array}\right\} \quad Z=\frac{(X-\mu)}{\sigma}
$$

1. Finding standard deviation of distribution requires using Cambridge Statistical Tables.

givent that $Z(\varphi=0.029)=1.9 \rightarrow Z=\frac{(4-0.504)}{\sigma}=1.9, \quad \sigma=1.84$

2. Probability that 2 independent fingerprints are equivalent, which means that the system recognizes at least 12 minutiae matches is determined as follows,

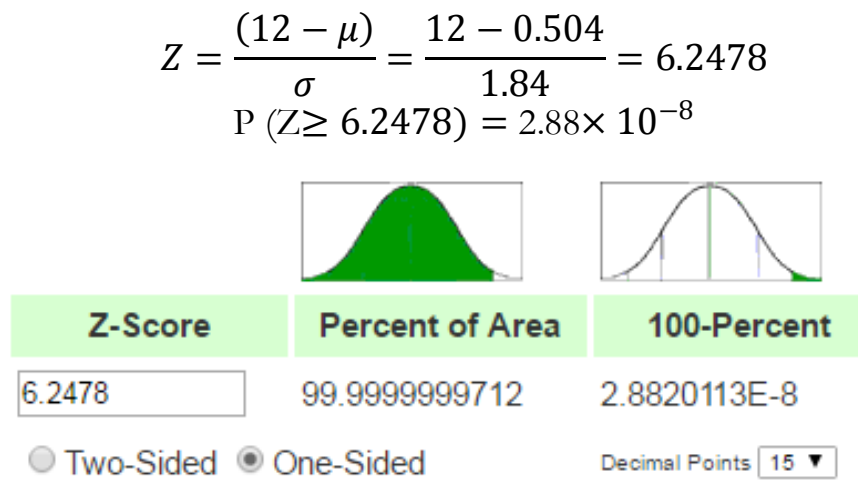


Figure 7. Calculation of percentiles.

Answer: the probability that the two fingerprints from different people are equivalent is $2.88 \times 10^{-8}$. Results show that the probability that their 12 minutiae points match is very close to zero, but it is still possible as it has 0.0000000288 chance of occurrence. If the world population exceeds $2.88 \times 10^{8}$ this amount, then occurrence of the same fingerprints is possible.

\subsection{Hypothesis Testing for the Reliability of Fingerprint Authentication System}

$H_{0}$ as a rule indicates no difference, and $H_{1}$ is the alternative hypothesis.

In the context of fingerprint authentication,

$\mathrm{H}_{0}: 2$ fingerprints from different persons can be equivalent.

$\mathrm{H}_{1}$ : 2 fingerprints from different persons cannot be equivalent.

$$
\begin{gathered}
\mathrm{H}_{0} \mu=\mu_{0} ; \mathrm{H}_{1} \mu \neq \mu_{0} \\
X \sim N(0,1) ; \sigma=1.84 ; E X=0.504
\end{gathered}
$$

Let us consider the conservative option of alternative hypothesis which leads to a 2 -sided test. First, test $\mathrm{H}_{0}$ at $5 \%$ significance level.

$$
\begin{gathered}
Z=\frac{(12-\mu)}{\sigma}=\frac{12-0.504}{1.84}=6.2478 \\
\alpha=0.05 \rightarrow Z_{\frac{\alpha}{2}}=z_{0.025}= \pm 1.96 \\
Z>z_{0.025} ; 6.2478>1.9600
\end{gathered}
$$

Next, test $\mathrm{H}_{0}$ at $1 \%$ significance level.

$$
\alpha=0.01 \rightarrow Z_{\frac{\alpha}{2}}=z_{0.005}= \pm 2.5758, \mathrm{Z}>z_{0.005}, 6.2478>2.5758
$$

We reject the null hypothesis at 5\% significance level and at $1 \%$ significance level, because $6.2478>2.5758>1.9600$. It indicates that the result is highly significant. So there's strong evidence to reject the null hypothesis, meaning that we can assume that alternative hypothesis is correct, that is 2 fingerprints cannot be equivalent. It does not necessarily indicate that the null hypothesis is wrong, but it shows that having the same fingerprint with somebody else is not likely to occur. However, the limitation of such test depends on how one structures the hypotheses. Consider,

H null: Fingerprint belongs to the person for which the test matches it. (Assumptions: Fingerprint scanner/system match correctly, fingerprint is unique).

$\mathbf{H}$ alternative: Fingerprint does not belong to the person for which system matches it. Assumptions: fingerprint is not unique, or system functions incorrectly.

Type 1 error is when Null Hypothesis is rejected, given that is true. It is also alpha, the size of the test. So alpha equals to the probability that fingerprint belongs to the person authenticating into the system, but system doesn't match it correctly (it either rejects or mismatches entry). It is similar to the False Rejection Rate.

Type 2 error is when Null Hypothesis is failed to reject, given that it is false. In other words, test performed by the system shows that fingerprints match, but in reality they don't belong to the same person. It is similar to False Match/Acceptance Rate used by biometric system. 
Additionally, using Poisson distribution we are able to find the probability of at least 22 minutiae points' matches, thus, making a match of two random fingerprints (Thumb, 2004). If $x=22$ minutiae points, the two fingerprints will identically match. $\lambda=0.83$. It is the expected value identified by experiment.

$$
P(x=22 ; \lambda=0.83)=\frac{e^{-\lambda} \lambda^{x}}{x !}=\frac{e^{-0.83} 0.83^{22}}{22 !}=6.43 \times 10^{-24}
$$

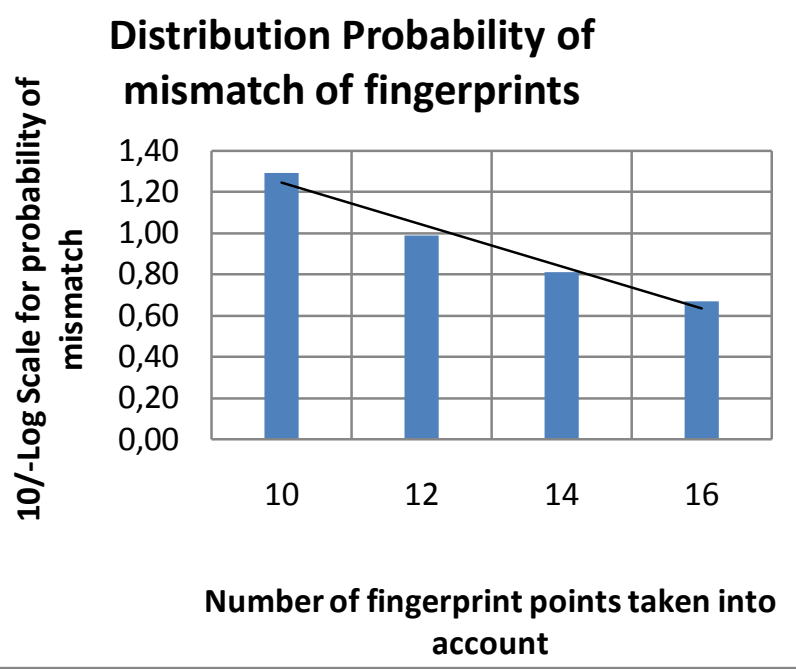

Figure 8. Distribution Probability of mismatch under Poisson distribution.

Consequently, according to Poisson distribution the probability of having the same 22 minutiae points with someone else is $6.43 \times 10^{-24}$. It is almost 0 . Hence, there's very low probability of having the same fingerprint with someone else, but it is possible if the number of people exceed this approximated value.

Using parameters of binomial distribution, we can calculate probability of mismatch, when $x=22$, extrapolating data from real-time experiment.
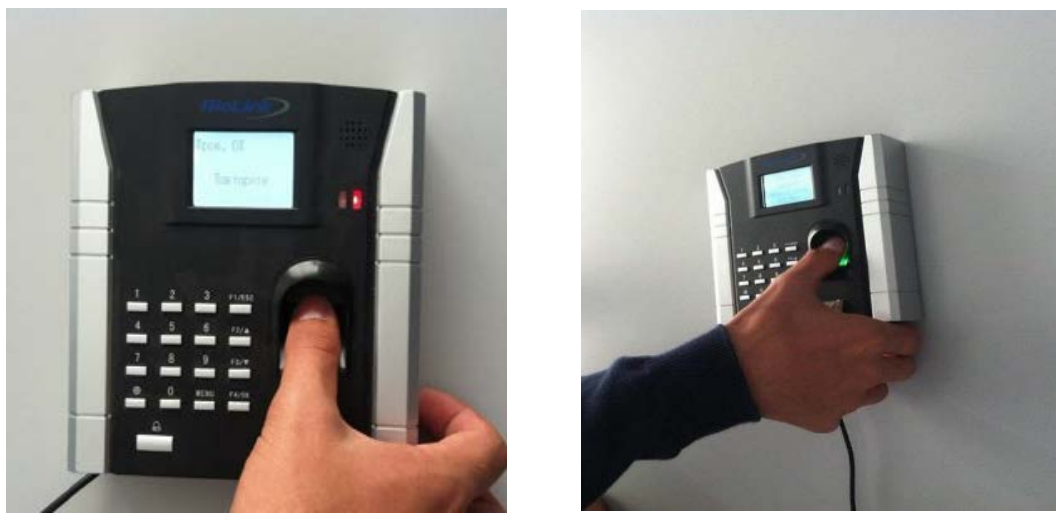

Figure 9. Experiment with Fingerprint Scanner. 


$$
\begin{gathered}
\frac{N(\text { matches })}{N(\text { trials })}=\frac{10}{12}=0.83=\pi \\
P(X=22)=0.83^{22}(1-0.83)^{0} C_{22}^{22}=0.0165 \approx 1.65 \%
\end{gathered}
$$

Secondary sources with 60000 operational activities with fingerprints resulted $90 \%$ rate of match with 1\% probability of False Acceptance Rate. So the experiment results and secondary data results show somewhat similar FAR values.

\subsection{Privacy And Social -Ethical Concerns Related to Fingerprint System}

The trust upon fingerprint technology is examined using survey below.

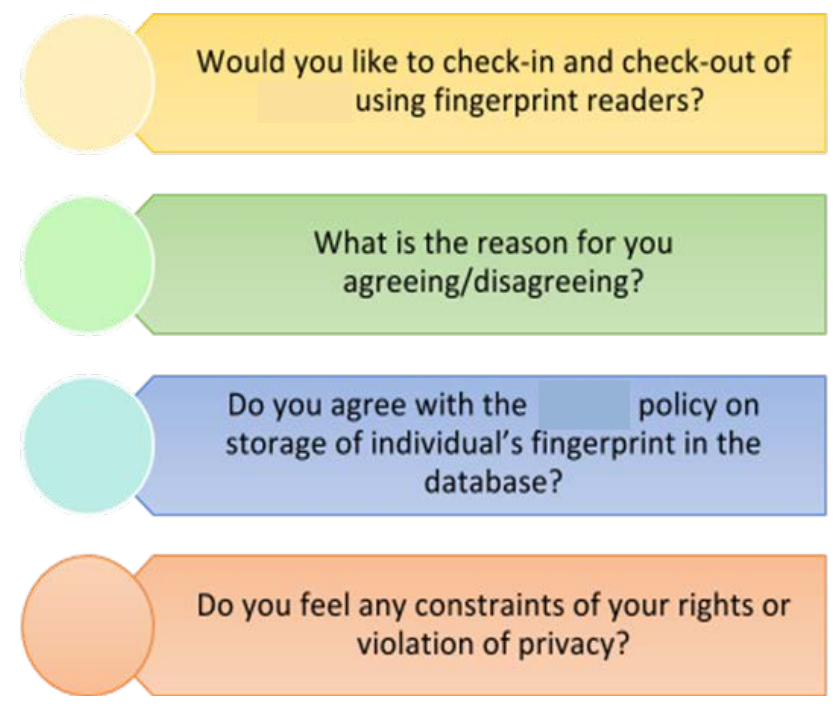

Figure 10. Survey for assessing trust upon fingerprint technology by stakeholders.
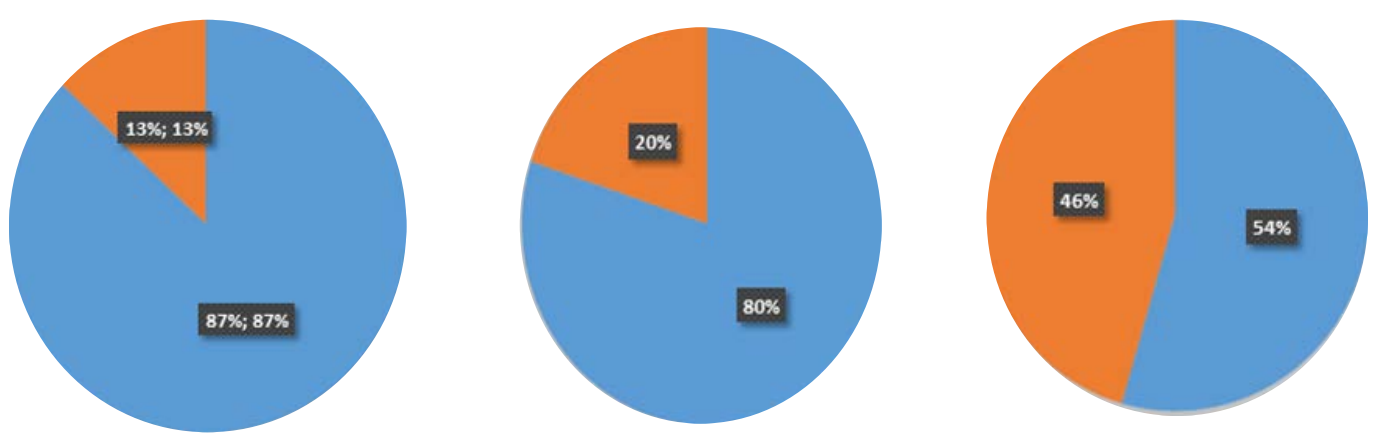

Figure 11. Diagram representing attitudes for/ against fingerprint identification for entrance system among a.adults, b.children, c.overall stakebolders.

For the first question, $13 \%$ of adult population is against entering company using their 
fingerprints. They are mainly foreign staffs that don't want to share their biometric data. There are also respondents who had a bitter experience using the fingerprint technology previously, because it doesn't function appropriately in cold weather. The majority of adults (blue shaded area) agree to enter company using fingerprints, because they consider fingerprint technology reliable. Unlike adults, children show the highest resistance for usage of fingerprint technology for authentication. They perceive it as 'too much control' over them, although majority prefer security over privacy.

$80 \%$ of surveyed stakeholders agree to use fingerprint technology for authentication into building. One of the primary reasons for supporting it is that fingerprints are always in your belongings; they cannot be lost or forgotten such as passwords, cards or tickets.

Further area of improvement is to test whether individual fingerprints are secure in the database by checking the encryption and quantization of fingerprint image into binary form as well as identifying who has an access to the database, where the binary images are stored. An issue concerning insecure storage of fingerprint data makes the use of biometrics technology not completely reliable. As biometrics expert Simo Huomio said, "Only with proper system design and smart use of strong cryptography biometric identification systems can claim their big promises. On worst scenarios possibility of wholly new kind of fraud is possible. This is due to the fact that biometric information of large amount of individuals stored on central databases is always a risk to our privacy (Huimo, n. d.)."

\section{Conclusions}

After research and development market for fingerprint technology in business has just dramatically expanded by 2016. According to performed survey stakeholders do not completely trust biometrics. However, development of well-designed fingerprint authentication system that considers more minutiae points for input data, that takes into account not only coordinates of minutiae points, but uses segments in modelling, that would function well in cold weather, and would not store the fingerprint image on system's database but only digitized values with limited access and strong IT security; trust towards fingerprint technology increases by stakeholders. It is already experiencing increasing demand, but could be improved. Because fingerprints are unique only until world population doesn't reach specific amount provided by statistical analysis above. Greater system accuracy will gain more trust and will stimulate higher usage rate by organizations and lead to greater environmental as well as economic sustainability.

Fingerprint Technology should not be applied in every possible way; it should protect insecure part of population, children, and some governmental bodies which work with confidential information. If it is applied within weak IT security and poor system design, third parties might gain access to fingerprint storage, which leads to crucial implications, where privacy of stakeholders is at risk. The application of fingerprint readers is environmentally friendly both in production and usage process with minimum consumption of resources. This way it contributes to sustainability in terms of resource efficiency, minimizes environmental impact compared to substitute systems' and meets the needs of operational efficiency. 


\section{References}

Biometrics.(n.d.) Competitive Landscape. Retrieved from http://biometrictechs.tripod.com/comp.html

Barinova, A. (2016). The First Country in the World to Deny Deforestation [article]. Retrieved from http://www.nat-geo.ru/nature/872172-pervaya-v-mire-strana-otkazavshayasya-ot-vyrubki-lesa/

Chang, L. (2016). Tourists in Japan could soon use their fingerprints as currency [article]. Retrieved from https://www.yahoo.com/tech/fingerprints-being-fingered-additional-applications175319291.html chang 2016

Fingerprint Technology Overview (n.d.). Identity One. Retrieved from $<$ http://www.identityone.net/BiometricTechnology.aspx >

Genetic Test. (2014). Dermatoglyphics. Retrieved from http://genetic-test.ru/dermatoglifika

Hawthorne, M. (2009). Fingerprints: Analysis and Understanding. Boca Raton, CRC Press

Huimo, S. (n.d.) Biometrics Identification. Retrieved from <http://www.tml.tkk.fi/Opinnot/Tik110.501/1998/papers/12biometric/biometric.htm>

Kothavalte, M., Markworth, R., Sandhu, P. (2004). Computer Security SS3. The University of Birmingham. Retrieved from

cs.bham.ac.uk/ mdr/teaching/modules03/security/students/SS3/handout/>

Meet, M. (2011) Plastic Cards Not So Fantastic for Environment [article]. Retreived from http://www.greenlivingtips.com/articles/greening-plastic-cards.html

Roethenbaugh, G. (1998). Biometrics Explained. Retrieved from <http://www.nsca.com/services/consortia/cbdc/explained.html>

Rowe, I. (2016). Simple, affordable technologies paving the way in sustainable business [article].Retrieved from https://blogs.unicef.org.uk/2016/04/06/simprints-sustainable-business/

Straub, M. (2016). Biometrics makes life easier and safer for customers - A South African case study. Retrieved from https://home.kpmg.com/xx/en/home/insights/2016/01/biometrics-makes-lifeeasier-and-safer-for-customers-fs.html

Tsai-Yang-Jea, Venu Govindaraju. (2005). A minutia-based partial fingerprint recognition system. Retrieved from http://citeseerx.ist.psu.edu/viewdoc/download?doi=10.1.1.211.9951\&rep=rep1\&type=pdf

Velandia, A., Katherine, J. (2012). Sustainable Development Based Innovation Technology in fare collection system [article]. content/uploads/abstracts/rio/selected/3624.pdf

Zhang, D. (2000). Automated Biometrics. Kluwer Academic Publishing, London. 